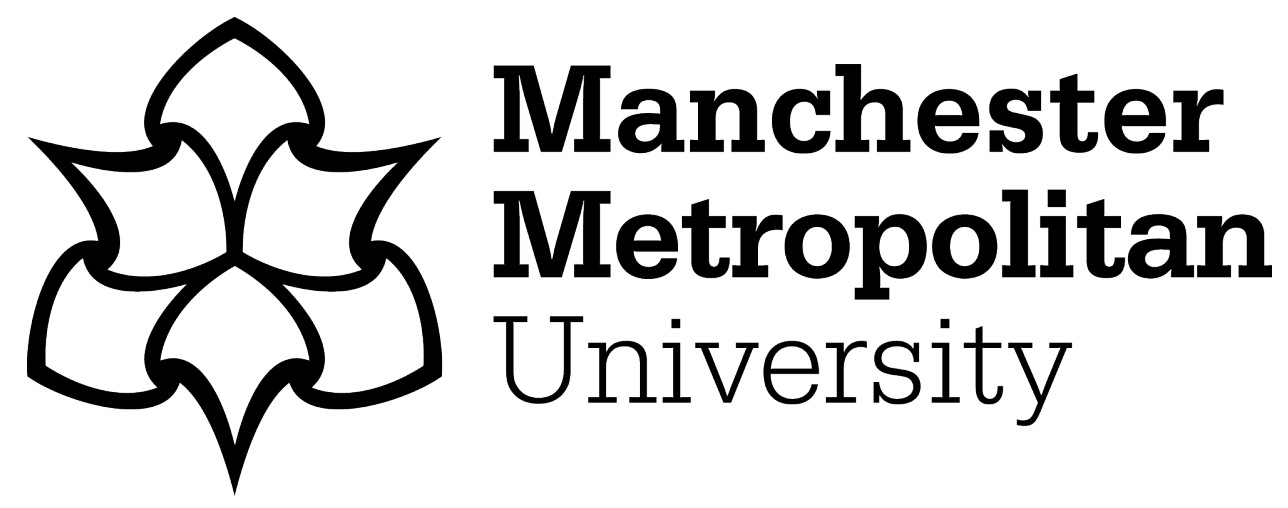

Hammoudeh, M and Arioua, M (2018) Sensors and actuators in smart cities. Journal of Sensor and Actuator Networks, 7 (1). ISSN 2224-2708

Downloaded from: https://e-space.mmu.ac.uk/620828/

Publisher: MDPI AG

DOI: https://doi.org/10.3390/jsan7010008

Usage rights: Creative Commons: Attribution 4.0

Please cite the published version 


\title{
Sensors and Actuators in Smart Cities
}

\author{
Mohammad Hammoudeh ${ }^{1, *,+}$ and Mounir Arioua ${ }^{2,+}$ \\ 1 School of Computing, Mathematics \& Digital Technology, Manchester Metropolitan University, \\ Manchester M15 6BH, UK \\ 2 National School of Applied Sciences, Abdelmalek Essaadi University, Tétouan 93000, Morocco; \\ m.arioua@uae.ma \\ * Correspondence: M.Hammoudeh@mmu.ac.uk; Tel.: +44-(0)161-247-2845 \\ + These authors contributed equally to this work.
}

Received: 9 February 2018; Accepted: 14 February 2018; Published: 16 February 2018

\section{Scope and Aim}

With the city, from its earliest emergence in the Near East between 4500 and 3100 BCE, came a wide range of new discoveries and inventions, from synthetic materials to wheeled vehicles. Through its dense population, irrigation, social continuity and physical security, emerged civil engineering, monumental construction, sculpture, mathematics, arts and law. Today, there is an enormous set of ideas and notions with respect to our ways of living, e.g., the ramp and the lever, which are still fundamental to cities' environmental, social, and economic structures. Modern-day smart cities compete for the introduction of smart technologies and applications to improve key areas of urban communities, such as system automation, sustainability, and quality of life. Technology research experts paint thrilling images of futuristic cities. What's glossed over, however, is the sensor and actuator technologies that enable these smart cities; in particular, the reliable, heterogeneous, wireless networks specifically designed to provision communication across a countless number of sensors embedded in almost everything.

The world is on the verge of a new epoch of innovation and change with the emergence of Wireless Sensor Networks (WSN). The convergence of smaller, more powerful processors, smart mobile devices, low-cost sensing, big data analytics, cloud hosting and new levels of connectivity allowed by the Internet is fuelling the latest wave of Machine-to-Machine (M2M) technology. The merits of this marriage of machines and the digital world are multiple and significant. It holds the potential to dramatically alter the way in which most global industries, such as buildings, rail transportation, power grids and healthcare operate on daily basis. WSNs expand to include our vehicles and homes, as well as newly developed wearable and implanted sensors, which brings fundamental transformations to many aspects of daily life.

WSN innovations promise to integrate and optimise smart buildings, autonomous vehicles, power grids, etc., to enable a successful transition towards smart, user-driven and demand-focused city infrastructures and services [1,2]. There is a wide range of current smart cities applications, which make our lives easier and more efficient, e.g., a smart phone application that let users find free parking spaces in the centre of town. However, cities are notoriously inefficient. As populations grow, everything from garbage collection and public transport becomes more expensive and complex. Away from increasing spending, there is also a demand from citizens for smarter services driven by sensorand actuator-based infrastructure.

In this Special Issue, we accepted submissions that focus on implementing intelligent sensing infrastructure to solve the smart cities conundrum. This Special Issue attracted contributions from academic researchers in computer science, communication engineering and physics, as well as information technology industry consultants and practitioners, in various aspects of sensors and 
actuators for smart cities. In the next section, we present a brief review of the papers published, highlighting their objectives and contributions.

\section{A Review of Contributions in this Special Issue}

Zhang et al. [3] address the challenge of large scale data analytics for smart cities. Typically, multi-modal sensor data collected from cyber physical environments, such as smart cities, must be processed before it can be can be used by data discovery, integration and mash-up protocols. With heterogeneous, noisy and incomplete data, clustering algorithms are used to organise the data in a dataset into clusters. The authors propose a new peak searching algorithm that uses Bayesian optimisation to find probability peaks in a dataset to increase the speed and accuracy of data clustering algorithms. This proposed clustering algorithm was thoroughly evaluated in simulation and results show that it significantly decreases the required number of clustering iterations (by 1.99 to 6.3 times), and produce clustering which, for a synthetic dataset, is 1.69 to 1.71 times more accurate than it is for traditional expectation-maximization (EM). Moreover, the algorithm correctly identified the outliers in a real dataset, decreasing iterations by approximately 1.88 times, while being 1.29 times more accurate than EM at a maximum.

Ateya et al. [4] contribution addresses the development of intelligent core network for Tactile Internet and future smart systems. Tactile Internet is an extremely low latency communication network with high availability, reliability and security. Tactile Internet is predicted to bring a new dimension to human-to-human and human-to-machine interaction in a multitude of different smart city aspects such transport, power grid, education, healthcare and culture. This paper presents a Tactile Internet system structure, which employs software defined networking in the core of the cellular network and mobile edge computing at multi-levels. The contribution focuses on the structure of the core network. The proposed system is simulated under reliable environmental conditions and results shows that it achieved a round trip latency of orders of $1 \mathrm{~ms}$ by the reducing the number of intermediate nodes that are involved in the communication process.

Jiang et al. [5] address the problem of establishing a good measure of the agreement between the activities detected from sensor-generated data and those recorded in self-reported data. The contribution reports on a trial conducted in three single-occupancy households from which data is collected from a set of sensors and from time use diaries completed by the occupants. The authors demonstrate the application of Hidden Markov Models with features extracted from mean-shift clustering and change points analysis. Then, a correlation-based feature selection is applied to reduce the computational cost. Finally, a method based on Levenshtein distance for measuring the agreement between the sensor-detected activities and that reported by the participants is demonstrated. The authors conclude their paper by an exciting discussion on lessons learnt on how the features derived from sensor data can be used in activity recognition and how they relate to activities recorded in time use diaries.

Taking the work in the previous contribution [5] one step further, Castro et al. [6] present a system based on the Internet of Things (IoT) to Human Activity Recognition (HAR) that monitors vital body signs remotely. The authors employ machine learning algorithms to determine activities that occur within four pre-defined categories (lie, sit, walk and jog). Evaluation using advanced real-world hardware platform shows that the proposed system is able to give feedback during and after the activity is performed, using a remote monitoring component with remote visualization and programmable alarms. This system was successfully implemented with a $95.83 \%$ success ratio.

The contribution of Riouali et al. [7] addresses another vital area of sensing and actuation in smart cities. The authors present a road traffic management system based on wireless sensor networks. This paper introduces the functional and deployment architecture of this system with particular focus on the data analytics component, which uses a new extension of batches Petri nets for modelling road traffic flow. The evaluation of the proposed system was performed using a real world implementation of visualization and data analysis components. 
Voutos et al. [8] present a social environmental sensor network integrated within a web GIS platform. Controls, user interface and extensions of the proposed system are presented. The key novel aspect of this contribution is the fact that the gathered data from the proposed system carries spatial information, which is fundamental for the successful correlation between pollutants and their place of origin. The latter is implemented by an interactive Web GIS platform operating oversight in situ and on a timeline basis.

Catania et al. [9] contribute a user-generated services composition in smart multi-user environments. In this contribution, the focus is on security issues raised by services generated by users, User-Generated Services (UGSs). UGSs are characterized by a set of features that distinguish them from conventional services. To cope with UGS security problems, the authors introduce three different policy management models, analysing benefits and drawbacks of each approach. Finally, a cloud-based solution that enables the composition of multiple UGSs and policy models, allowing users' devices to share features and services in IoT based scenarios is proposed.

Obinikpo et al.'s [10] contribution demonstrates how big sensed data meets deep learning for smarter health care in smart cities. Healthcare lends itself as a natural fit for IoT technology and smart city concepts. The authors advocate that integrating sensory data (hard sensing) with external data sources (soft sensing, e.g., crowd-sensing) could reveal new data patterns and information. This research addresses this challenge through hidden perception layers in the conventional artificial neural networks, namely by deep learning. The paper starts by reviewing deep learning techniques that can be applied to sensed data to improve prediction and decision making in smart health services. Then, a comparison and taxonomy of these methodologies based on types of sensors and sensed data is presented. Finally, a thorough discussions on the open issues and research challenges in each category is given.

Arbia et al.'s [11] contribution targets smart city critical infrastructure, particularly, an IoT enabled end-to-end emergency and disaster relief system. This paper presents a new enhancement for an emergency and disaster relief system called Critical and Rescue Operations using Wearable Wireless sensors networks (CROW 2). CROW 2 addresses the reliability challenges in setting up a wireless autonomous communication system to offload data from the disaster area (rescuers, trapped victims, civilians, media, etc.) back to a command centre. The proposed system connects deployed rescuers to extended networks and the Internet. The system integrates heterogeneous wireless devices and different communicating technologies to enable end-to-end network connectivity, which is monitored by a cloud-based IoT platform. The overall performance of CROW 2 is evaluated using end-to-end link quality estimation, throughput and end-to-end delay. Finally, the system architecture is validated through deployment and motion detection and links unavailability prevention are highlighted.

\section{Conclusions and Remarks}

Sensors and actuators are the building blocks for the forth industrial revolution. They have already transformed the way humans perceive their environment. Sensor-enabled smart cities are paving the way for a more sustainable future, from urban planning to social conscience. The papers published in this special issue put humans at the centre of smart cities. From traffic management to assisted living, human centred design of smart city services is a detrimental factor to the success of smart cities. It is evident that we are still at the start of the smart cities revolution and the full economical, environmental and social benefits are yet to be achieved.

Conflicts of Interest: The authors declare no conflict of interest.

\section{References}

1. Coates, A.; Hammoudeh, M.; Holmes, K.G. Internet of Things for Buildings Monitoring: Experiences and Challenges. In Proceedings of the International Conference on Future Networks and Distributed Systems, ICFNDS '17, Cambridge, UK, 19-20 July 2017; ACM: New York, NY, USA, 2017. 
2. Jogunola, O.; Ikpehai, A.; Anoh, K.; Adebisi, B.; Hammoudeh, M.; Son, S.Y.; Harris, G. State-of-the-art and prospects for peer-to-peer transaction-based energy system. Energies 2017, 10, 2106.

3. Zhang, T.; Zhao, Q.; Shin, K.; Nakamoto, Y. Bayesian-Optimization-Based Peak Searching Algorithm for Clustering in Wireless Sensor Networks. J. Sens. Actuator Netw. 2018, 7, 2.

4. Ateya, A.A.; Muthanna, A.; Gudkova, I.; Abuarqoub, A.; Vybornova, A.; Koucheryavy, A. Development of Intelligent Core Network for Tactile Internet and Future Smart Systems. J. Sens. Actuator Netw. 2018, 7, 1.

5. Jiang, J.; Pozza, R.; Gunnarsdóttir, K.; Gilbert, N.; Moessner, K. Using Sensors to Study Home Activities. J. Sens. Actuator Netw. 2017, 6, 32.

6. Castro, D.; Coral, W.; Rodriguez, C.; Cabra, J.; Colorado, J. Wearable-Based Human Activity Recognition Using and IoT Approach. J. Sens. Actuator Netw. 2017, 6, 28.

7. Riouali, Y.; Benhlima, L.; Bah, S. Extended Batches Petri Nets Based System for Road Traffic Management in WSNs. J. Sens. Actuator Netw. 2017, 6, 30.

8. Voutos, Y.; Mylonas, P.; Spyrou, E.; Charou, E. A Social Environmental Sensor Network Integrated within a Web GIS Platform. J. Sens. Actuator Netw. 2017, 6, 27.

9. Catania, V.; La Torre, G.; Monteleone, S.; Panno, D.; Patti, D. User-Generated Services Composition in Smart Multi-User Environments. J. Sens. Actuator Netw. 2017, 6, 20.

10. Obinikpo, A.A.; Kantarci, B. Big Sensed Data Meets Deep Learning for Smarter Health Care in Smart Cities. J. Sens. Actuator Netw. 2017, 6, 26.

11. Ben Arbia, D.; Alam, M.M.; Kadri, A.; Ben Hamida, E.; Attia, R. Enhanced IoT-Based End-To-End Emergency and Disaster Relief System. J. Sens. Actuator Netw. 2017, 6, 19.

(C) 2018 by the authors. Licensee MDPI, Basel, Switzerland. This article is an open access article distributed under the terms and conditions of the Creative Commons Attribution (CC BY) license (http:/ / creativecommons.org/licenses/by/4.0/). 